Research Article

\title{
SISTEMA CONVECTIVO DE MESOESCALA OBSERVADO NO DIA 12 DE DEZEMBRO DE 1992 DURANTE TOGA-COARE: ESTRUTURA CI- NEMÁTICA E TRANSPORTE DE QUANTIDADE DE MOVIMENTO
}

\author{
C. J. de Campos ${ }^{1} \&$ M. Chong ${ }^{2}$
}

No dia 12/12/92, durante o experimento de campo TOGA-COARE (Tropical Ocean Global Atmosphere-Coupled Ocean-Atmosphere Response Experiment) uma missão foi efetuada, detectando uma região de convecção a NE da zona chamada de "Intensive Flux Array " (IFA: $1^{\circ} \mathrm{N}-4^{\circ} \mathrm{S}, 151-158^{\circ} \mathrm{E}$ ). Essa região caracterizava-se por apresentar uma circulação ciclônica nos baixos níveis e um fluxo divergente de NE em altitude, caracterizando a atividade convectiva do sistema. A análise das imagens no canal infravermelho do satélite GMS-4 indicava uma discreta propagação do sistema em direção a sudoeste. Os dois aviões WP-3D da "National Oceanic and Atmospheric Administration" (NOAA), utilizando a técnica de varredura FAST (Fore/Aft Scanning Technique), descreveram trajetórias paralelas coordenadas, primeiramente ao longo da linha convectiva e em seguida dentro da região estratiforme do sistema. Os dados de radares Doppler coletados durante essa missão mostravam a existência de células convectivas moderadas, localizadas dentro de uma área de precipitação estratiforme, e células convectivas mais intensas localizadas a sudoeste do sistema. A combinação das porções paralelas das trajetórias de cada avião foi utilizada para restituir o campo de vento 3D, usando a técnica variacional EODD (Extended Overdetermined Dual-Doppler). O campo de vento 3D, derivado dos dados de radar, serviu para estudar as características do sistema observado e para examinar a estrutura dos transportes de quantidade de movimento operado pelo sistema. A estrutura cinemática do sistema mostrou características clássicas, com um fluxo ascendente na frente do sistema que transporta o ar das camadas inferiores para o alto e um fluxo descendente atrás da linha convectiva. Os resultados para os transportes de quantidade de movimento também mostraram características similares a outros casos estudados anteriormente, tais como: (i) o transporte vertical de quantidade de movimento horizontal perpendicular à linha convectiva é amplificador do gradiente, resultando no aumento do seu cisalhamento vertical; (ii) para a componente paralela (v), a divergência vertical do fluxo v reduziu o seu cisalhamento vertical, gerando um transporte redutor do gradiente.

Palavras-chave: Sistema convectivo de mesoescala; Transporte de quantidade de movimento; Técnica EODD; TOGA-COARE.

MESOSCALE CONVECTIVE SYSTEM OBSERVED ON DECEMBER 12, 1992 DURING TOGA-COARE: KINEMATIC STRUCTURE AND MOMENTUM TRANSPORT-On December 12 1992, during TOGA-COARE (Tropical Ocean Global Atmosphere - Coupled Ocean-Atmosphere Response Experiment), enhanced convection was observed in the northeast of the COARE Intensive Flux Array (IFA: $I^{\circ} \mathrm{N}-4^{\circ} \mathrm{S}, 151-158^{\circ} \mathrm{E}$ ), occurring in a broad cyclonic flow at low levels and divergent northeasterly flow at upper levels. Satellite IR (Infra Red) pictures revealed scattered Class 2/3 convection, and indicated a discrete propagation toward southwest. Radar observations from two NOAA WP-3D aircrafts showed moderated convective cells, located in a stratiform precipitation region, and stronger convective cells in the southwest part of the system. During the two-plane mission, several "quad-Doppler" observations were obtained from the four-pattern legs described by each aircraft, first along the convective line and after in the stratiform part of the system. Combination of parallel portions of these aircraft flight tracks was made to synthesize the $3 D$ wind fields, using the Extended Overdetermined Dual-Doppler technique (EODD). These radar-derived wind fields serve to highlight the main characteristics of the observed 


\section{Mesoscale Convective System}

system and to examine the momentum transports occuring in the system. The derived airflow structure shows classical characteristics, with an updraft flow in front of the system transporting low-level air to high levels and a downdraft airflow in the rear. The results to the momentum transports reveals that this system presents similar features of previously observed ones, such as: (i) the vertical transport of horizontal momentum normal to the convective line is countergradient resulting in a increase of its vertical shear; (ii) the vertical transport of line-parallel wind $(v)$ is downgradient because the vertical divergence of the vertical v-momentum flux decreases the $v$ shear.

Key words: Mesoscale convective system; Momentum transport; EODD technique; TOGACOARE.

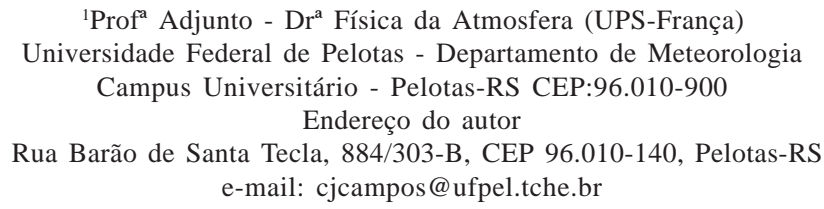

${ }^{2}$ Diretor de Pesquisas CNRS-CNRM - Dr. Física da Atmosfera (Universidade de Paris - França) Centre National de Recherches Météorologiques Toulouse - France

\section{INTRODUÇÃO}

Sistemas Convectivos de Mesoescala (SCM), que ocorrem freqüentemente nos trópicos e nas latitudes médias, têm sido objeto de vários estudos por pesquisadores da área de ciências atmosféricas. Para o estudo dos SCM, a utilização de radares Doppler embarcados em aviões é primordial, pois estes permitem o acesso ao ciclo de vida dos sistemas, graças a sua mobilidade. Neste sentido, o experimento de campo TOGA-COARE (Tropical Ocean Global Atmosphere-Coupled OceanAtmosphere Response Experiment; Webster \& Lukas, 1992) utilizou radares Doppler embarcados nos dois aviões WP-3D da NOAA (National Oceanic and Atmospheric Administration). Esses radares estavam equipados com a técnica FAST (Fore/Aft Scaning Technique; Jorgensen \& DuGranrut, 1991) e foram essenciais para os objetivos científicos de COARE, por fornecerem uma descrição detalhada da circulação e da precipitação para estudos diagnósticos de processos físicos que ocorrem nos SCM.

Dentro deste contexto, o presente trabalho teve por objetivo examinar a estrutura cinemática e os transportes de quantidade de movimento do SCM observado no dia 12 de dezembro de 1992, durante o experimento TOGA-COARE. Para tal, inicialmen- te o campo de vento 3D do SCM foi reconstituído pelo método EODD (Extended Overdetemined Dual-Doppler formalism) proposto por Chong \& Campos (1996), utilizando os dados coletados pelos radares Doppler embarcados nos dois aviões WP3D operados pela NOAA. Um campo de vento 3D composto foi obtido para fornecer uma visão global e sintética do sistema, sendo em seguida utilizado para calcular o transporte vertical de quantidade de movimento horizontal do SCM em estudo.

\section{OBSERVAÇÕES E PROCESSAMENTO DOS DADOS}

\section{Contexto sinótico}

Durante o experimento TOGA-COARE, foi observado a ocorrência de um SCM, no dia 12/12/ 92, localizado a nordeste da zona Intensive Flux Array (IFA: $1^{\circ} \mathrm{N}-4^{\circ} \mathrm{S}, 151-158^{\circ} \mathrm{E}$ ). Uma descrição da situação em grande escala deste sistema, utilizando campos de vento das 18:00 UTC do dia 12/ 12/92 e das 00:00 UTC do dia 13/12/92, em 850 e $200 \mathrm{hPa}$, gerados pelo modelo do CEP ("Centre Européen pour les Prévisions Météorologiques à Moyen Terme") foi feita por Campos (1996). Os campos de vento analisados mostraram uma circulação ciclônica nos baixos níveis e um fluxo 
divergente de NE em altitude, caracterizando a atividade convectiva do sistema.

Utilizando observações do satélite GMS-4 no canal infravermelho, Velden \& Young (1994) detectaram a ocorrência de convecção intensa a NE da IFA que se deslocava para oeste no período de 10 a 13 de dezembro. O aglomerado convectivo observado no dia 12 de dezembro fez parte de um mesmo sistema que pôde ser seguido entre os dias 11 e 13 de dezembro pelas imagens de satélite.

Analisando dados coletados pelo radar Doppler ventral embarcado no avião WP-3D N43RF da NOAA e imagens de satélite, Chen et al. (1996) observaram que o sistema varrido era um intenso e típico SCM oceânico. Este sistema apresentava uma região com precipitação convectiva, na sua porção sudoeste, e traços de uma região com precipitação estratiforme, na sua porção nordeste. Este sistema foi descrito por Redelsperger et al. (1995) como uma linha de instabilidade de orientação NO-SE, formada no dia 12 à frente de um aglomerado observado no dia 11 e em fase de dissipação no dia 12, que se deslocava para $\mathrm{SO}\left(238^{\circ}\right)$ com uma velocidade de $11 \mathrm{~m} \cdot \mathrm{s}^{-1}$, no período de 17:30 a 21:30UTC. Esta linha se transformou em seguida num aglomerado, antes de enfraquecer progressivamente, até dissipar-se no início da tarde do dia 13. Nova convecção iniciou-se ao longo da linha convectiva, na vizinhança do SCM dissipado do dia 12 e se deslocou para NE intensificando-se. Esta linha de instabilidade não foi reproduzida pela análise do CEP, apesar do traço de um aglomerado em fase dissipativa ter sido detectado. Uma simulação deste caso foi efetuada, inicializada à partir do campo de vento analisado de grande escala, para compreender o modo de geração da linha (Redelsperger et al., 1995).

As condições do ambiente para este sistema foram definidas em Campos (1996) utilizando dados de sondagem. A sondagem efetuada as 17:00 UTC do dia $12 / 12 / 92$ em Kapingamarangi $\left(154,80^{\circ} \mathrm{E}, 1,072^{\circ} \mathrm{N}\right)$, à oeste do sistema convectivo, apesar de ser um pouco afastada, mostrava um ambiente moderadamente instável. A CAPE (Convective Available Potential Energy) que fornece uma medida direta da energia potencial disponível para o desenvolvimento da atividade convectiva, estava em torno de $700 \mathrm{~J} \cdot \mathrm{kg}^{-1}$. Quanto à estrutura do vento, foi observado um cisalhamento nos baixos níveis (abaixo de $1,5 \mathrm{~km}$ ) de $4,2 \mathrm{~s}^{-1}$, orientado para $238^{\circ}$, o que permitiu classificar este sistema como alinhado perpendicularmente ao cisalhamento do vento.

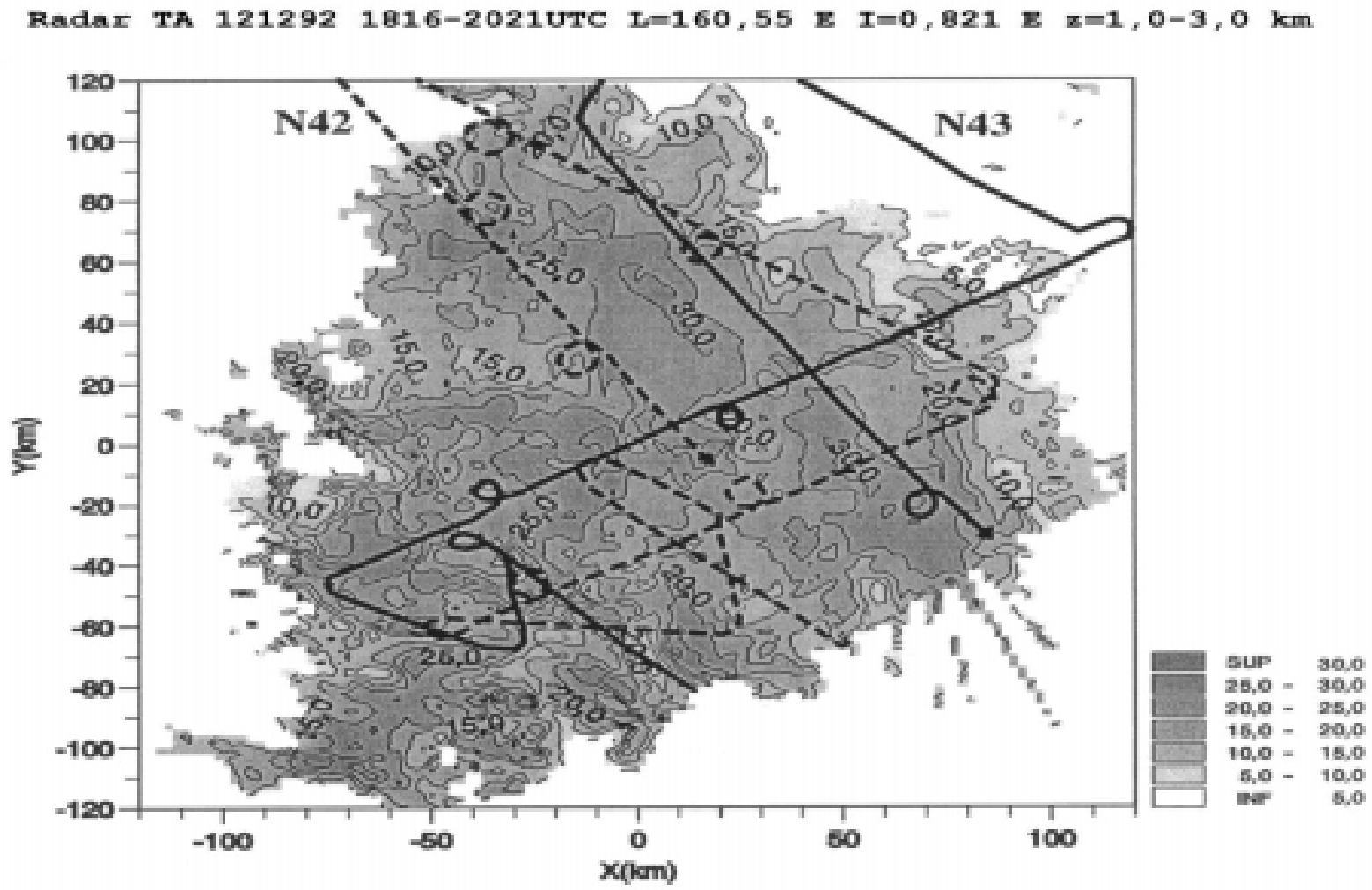

Figura 1- Campo de refletividade composto advectado, obtido pelo radar Doppler N43, para o período 18:16-20:21 UTC, num domínio de $240 \mathrm{~km}$ por $240 \mathrm{~km}$. As trajetórias dos dois aviões, N42 e N43, são também mostradas (respectivamente, as linhas pontilhadas e contínuas).

Figure 1- Composite reflectivity pattern from the $43 R F$ tail radar, for the period 18:16-20:21 UTC in a domain of $240 \mathrm{~km}$ by $240 \mathrm{~km}$. The faur pattern legs from $42 R F$ and $43 R F$ aircrafts are also shown (respectively, dashed and continuous lines). 


\section{Dados de radar}

No dia 12/12/92, entre 17:00 e 23:00 UTC, os dois aviões WP-3D da NOAA, equipados com radares Doppler, participaram de uma missão que varreu o sistema, usando a técnica de varredura "Fore/Aft Scaning Technique" (Jorgensen \& DuGranrut, 1991). Esta técnica tem por objetivo fazer os aviões descreverem trajetórias paralelas coordenadas, a fim de obter até 4 medidas de velocidade radial e refletividade radar em cada ponto do domínio varrido. Uma série de trajetórias (ida e volta) relativamente curtas (40 à $80 \mathrm{~km}$ ) foi primeiro executada dentro da região convectiva entre 17:50 e 18:30 UTC, antes de explorar a zona estratiforme do sistema. Esta varredura ocorreu durante a fase madura/dissipação do sistema. A Fig. 1 representa uma imagem do campo de refletividade radar, obtida pelo radar Doppler embarcado no avião P3N43, para um período de aproximadamente 2 horas (entre 18:16 e 20:21 UTC), numa camada entre 1 e 3 $\mathrm{km}$ de altitude. Esta imagem está centrada em $0,821^{\circ} \mathrm{N}$ e $160,55^{\circ} \mathrm{E}$ e cobre um domínio de $240 \times 240 \mathrm{~km}^{2}$. Para obter esta imagem cada posição do avião foi recalculada em relação à velocidade de advecção do SCM (11 m.s $\left.{ }^{1}\right)$ e ao tempo de referência 18:45 UTC, a fim de minimizar os efeitos temporais devido ao deslocamento do sistema para sudoeste. Essas posições estão representadas na Fig. 1 pela trajetória do avião N43 (linha contínua), que voava à $350 \mathrm{~m}$. À esta imagem foi sobreposta a trajetória do avião N42 (linha pontilhada) que voava a uma altura média de $4200 \mathrm{~m}$. O ângulo de rotação da antena era de $\sim 10 \mathrm{rpm}$ e a velocidade relativa ao solo do avião era de $\sim 120 \mathrm{~m} . \mathrm{s}^{-1}$, gerando uma resolução dos dados ao longo da trajetória de $\sim 1400 \mathrm{~m}$. A taxa de rotação da antena era equivalente a um incremento de 1,3 graus, enquanto o espaçamento dos dados na direção radial era de $150 \mathrm{~m}$, com um intervalo máximo de $38,5 \mathrm{~km}$. Os laços efetuados ao longo destas trajetórias em forma de quatro, são adaptados para extrair um perfil de vento médio, do tipo VADVelocity Azimuth Display (Browning \& Wexler, 1968). Os dados coletados durante a execução destes laços necessitam de um tratamento especial e não foram considerados neste estudo.

A Fig. 1, fornece uma visão global do sistema, mostrando uma zona constituída por células convectivas (atingindo $30 \mathrm{dBZ}$ ) na parte sudoeste do domínio, organizadas ao longo de um eixo NO-SE, que é seguida por uma região de precipitação estratiforme, que se estende por mais de $100 \mathrm{~km}$. Essa estrutura foi também observada por Chen et al. (1996). Uma seção vertical em $\mathrm{y}=-50 \mathrm{~km}$ (entre 18:15-18:30 UTC) mos- trou que a extensão vertical das células convectivas atingia até $15 \mathrm{~km}$ (não apresentado aqui).

\section{Restituição do campo de vento 3D pelo método EODD}

Neste trabalho os dados de radar (velocidade radial e refletividade radar), coletados durante a missão do dia 12/12/92, foram utilizados para restituir o campo de vento 3D do sistema convectivo, utilizando a técnica EODD (Chong \& Campos, 1996).

O método EODD é uma extensão do método ODD (Overdetermined Dual Doppler), ao qual foi adicionado duas condições limitantes, com o objetivo de controlar: (a) as variações de $u$ e $v$ a cada passo do processo iterativo utilizado para resolver o sistema de equações para $u$, ve $w$; (b) as variações horizontais do campo de vento horizontal quando este é mal determinado nas regiões, acima e abaixo do avião no método dual-Doppler.

O formalismo matemático utilizado no método EODD é escrito de tal sorte que a função $F$ seja mínima, onde F é dada por:

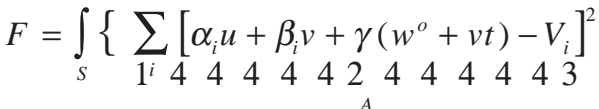

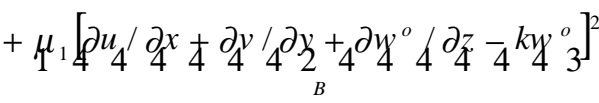

$$
\begin{aligned}
& \left.+\mu_{1}\left[J_{2}\left(u_{2}\right)+{ }_{C} J_{4}\left(v_{3}\right)\right]\right\} d x d y
\end{aligned}
$$

onde $\alpha, \beta$ e $\gamma$ são funções dos ângulos de elevação e azimute da antena do radar, u e v são as velocidades horizontais, $\mathrm{w}^{\mathrm{O}}$ é a velocidade vertical (especificada no início do processo iterativo e obtida da equação da continuidade em seguida), vt é a velocidade de queda, $V$ é a velocidade radial, $\mu_{1}$ é um fator de normalização, $\mu_{2}$ é um filtro proporcional ao comprimento de onda de corte, $\mathrm{k}=-\partial \ln \rho / \partial \mathrm{z}$ e $\mathrm{J}_{2}$ é um termo que minimiza as derivadas segundas do campo de u e v.

O termo A desta equação representa o método ODD, ele faz o ajuste de $\mathrm{u} e \mathrm{v}$ às medidas de velocidade radial.

O termo B limita as variações de u e v de uma iteração a outra, assim a contaminação de u e v devido aos erros na estimativa de w na integração da equação de continuidade é moderado.

Enfim, o termo $\mathrm{C}$ é um filtro que é controlado pelo peso $\mu_{2}$, que é proporcional ao comprimento de onda de corte. Ele regulariza o campo de u e v principalmente nas regiões de indeterminação, por exemplo, acima e abaixo do avião, quando somente duas medi- 
das são disponíveis.

A discretização da função $\mathrm{F}$ é feita em cada ponto de grade $\mathrm{k}=(\mathrm{j}-\mathrm{i}) \mathrm{n}_{\mathrm{x}}+\mathrm{i}$, onde $\mathrm{i}$ varia de 1 a $\mathrm{n}_{\mathrm{x}}$ ao longo do eixo x e j varia de 1 a $\mathrm{n}_{\mathrm{y}}$ ao longo do eixo y. A minimização de Fé em seguida obtida, derivando F em relação a $u_{k}$ e $v_{k}$ procurados, ou seja: $\partial F / \partial u_{k}=0$ e $\partial F /$ $\partial \mathrm{v}_{\mathrm{k}}=0$ onde $\mathrm{k}=1, \mathrm{n}_{\mathrm{x}} \mathrm{n}_{\mathrm{y}}$ conduzindo a um sistema linear $2 \mathrm{n}_{\mathrm{x}} \mathrm{n}_{\mathrm{y}}$ de equações, que pode ser escrito sob a forma matricial seguinte: $\overline{\bar{M}} \cdot \bar{V}=\bar{P}$, onde $\overline{\bar{M}}$ é a matriz de minimização, função das coordenadas das observações, $\bar{V}$ é o vetor dos coeficientes procurados e $\bar{P}$ é o vetor de minimização que depende das medidas.

A inversão deste sistema é efetuada de maneira iterativa, utilizando o método dos gradientes conjugados. A componete w é então obtida por integração da equação de continuidade, utilizando o método variacional desenvolvido por Chong \& Testud (1983).

Antes de aplicar o método EODD os dados foram corrigidos pelos efeitos de velocidade Doppler ambígua e pelos erros de navegação. O domínio de restituição utilizado foi um sub-domínio daquele representado na Fig. 1 com a mesma origem, ou seja, de 150 $\mathrm{km} \times 150 \mathrm{~km}$ x $14 \mathrm{~km}$, centrado em $0,821^{\circ} \mathrm{Ne} 160,55^{\circ} \mathrm{E}$. As observações feitas neste domínio foram igualmente corrigidas da advecção, sendo reposicionadas num sistema que se deslocava com a velocidade do SCM (11 m.s. $\mathrm{s}^{-1}$ para SO) e em seguida interpoladas numa grade cartesiana, com uma resolução horizontal e vertical de 2,0 e 0,5 km, respectivamente. O esquema de interpolação de Cressman (1959) foi utilizado, com a função peso tendo um raio de influência horizontal e vertical de 2,5 e 1,2 km, respectivamente.

Para estudar a estrutura cinemática deste sistema, os dados de radar das porções de trajetória varridas pelo avião P3-N42 foram combinadas com aquelas provenientes do radar embarcado no avião P3-N43. Os diferentes períodos de observações que formaram os pares de trajetórias para a análise das diferentes zonas do sistema convectivo foram: (i) região convectiva: 18:16-18:28UTC (P3-N42) e 18:1618:26UTC (P3-N43); (ii) parte das regiões convectiva e estratiforme: 18:45-19:13UTC (P3-N42) e 18:4519:15UTC (P3-N43); (iii) região estratiforme: 19:5020:21 UTC (P3-N42) e 19:52:20:13 UTC (P3-N43). Em seguida três campos de vento $3 \mathrm{D}$, foram obtidos aplicando-se o método EODD, às observações de cada par de trajetórias definidas acima. Esses campos de vento serão referidos a partir de agora como 1816, 1845 e 1950. A análise individual de cada um destes campos de vento, em nenhum momento forneceu uma observação global do sistema. A análise das 1845 foi a

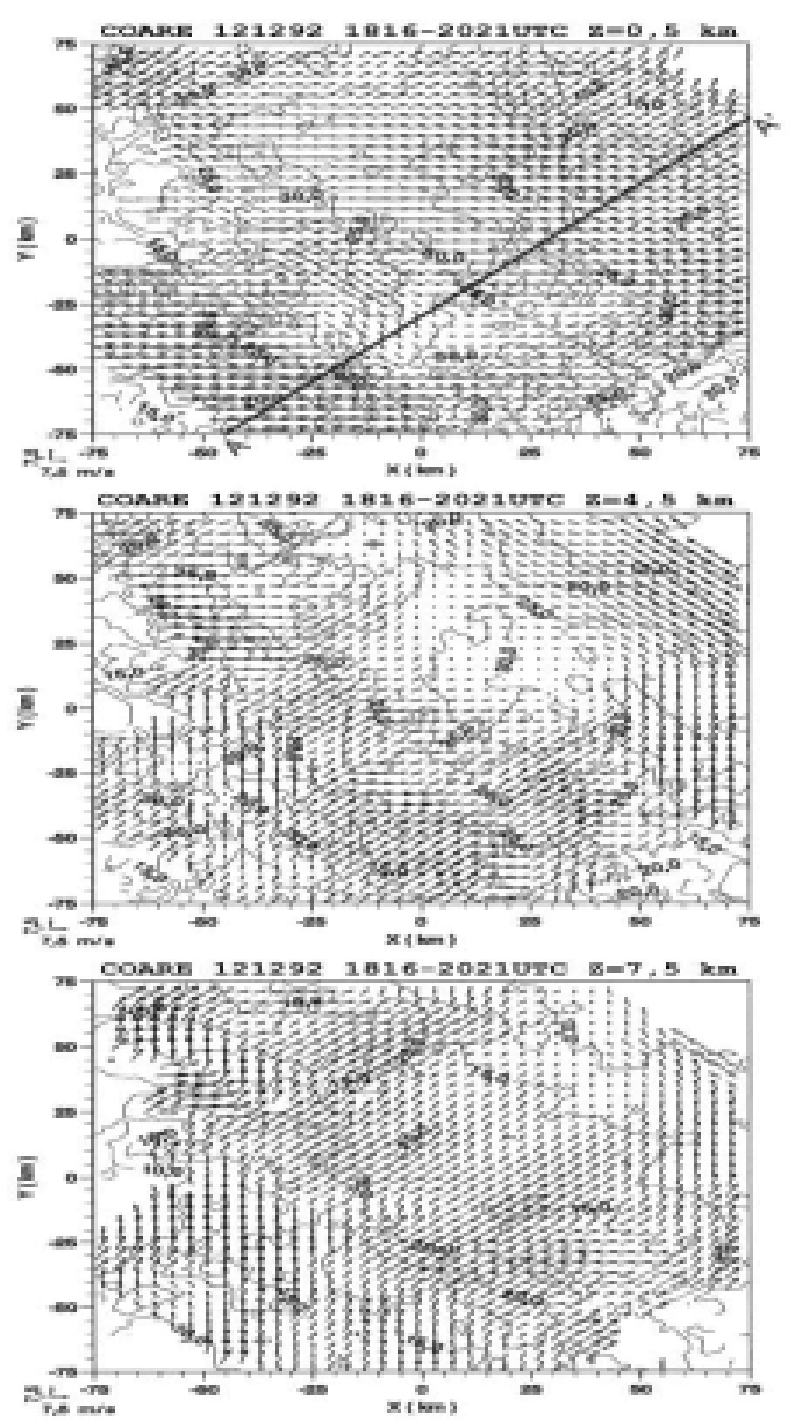

Figura 2- Fluxo horizontal relativo e isolinhas de refletividade em $\mathrm{z}=0,5,4,5$ e 7,5 km, para campo de vento composto. O norte é ao longo do eixo Y e a origem do domínio analisado é $160,55^{\circ} \mathrm{E}, 0,821^{\circ} \mathrm{N}$. Os vetores são traçados a cada $4 \mathrm{~km}$, com escala mostrada embaixo a esquerda.

Figure 2- Relative flow and reflectivity contours at $z=0.5,4.5$ and $7.5 \mathrm{~km}$, for the composite wind field. North is along y axis and origin of the analysed domain is at $160.55^{\circ} \mathrm{E}, 0.821^{\circ} \mathrm{N}$. Contour lines are radar reflectivity. Vectors are plotted every $4 \mathrm{~km}$, with scaling at the lower left corner.

que cobriu a maior parte do sistema, apresentando ainda assim, duas zonas não cobertas, ao sul e ao noroeste do domínio. Estas zonas foram, por outro lado, documentadas nas análises das 1816 e 1950. Assim foram combinados os três campos analisados para obter um campo composto, o que permitiu aumentar a zona de observação em todos os níveis e a realização de um estudo de impacto global. O campo de vento composto foi obtido a partir da média aritmética das componentes do vento em cada nível, dos campos 1816, 1845 e 1950 . 


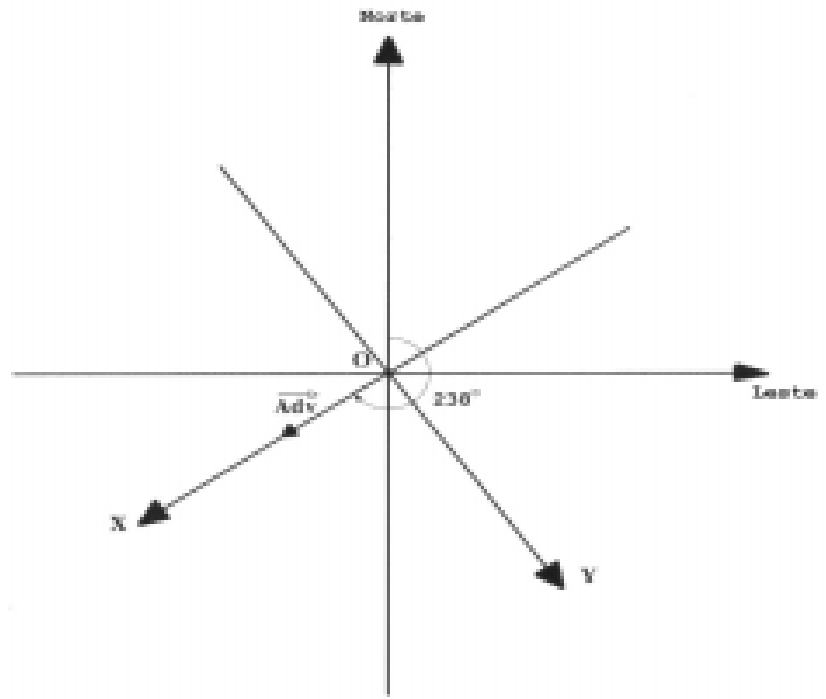

Figura 3- Posição do referencial $(\mathrm{O}, \mathrm{x}, \mathrm{y})$ da linha convectiva, em relação ao referencial norte-sul. Este referencial é orientado de modo que a componente $u$, perpendicular à linha convectiva, é positiva na direção de deslocamento da linha (vetor Adv).

Figure 3-Convective line coordinate system $(O, x, y)$. It is righthanded, with U normal to the convective line, positive in the direction of the line movement (Adv. vector).

\section{Cálculo dos transportes de quantidade de movimento}

Para calcular os transportes de quantidade de movimento, utilizou-se o campo de vento composto mostrado na Fig. 2.

A Teoria do Comprimento de Mistura (TCM) é freqüentemente utilizada para descrever o transporte vertical de quantidade de movimento horizontal pelos sistemas convectivos. Essa teoria supõe que o fluxo vertical de quantidade de movimento horizontal $\left(\bar{\rho} \overline{u^{\prime} w^{\prime}}\right)$, por exemplo, ocorre no sentido contrário ao gradiente vertical de velocidade, e age de maneira a diminuir o cisalhamento vertical do vento horizontal, modificando a quantidade de movimento horizontal e produzindo uma mistura com o ambiente. Matematicamente tem-se $\bar{\rho} \overline{u^{\prime} w^{\prime}}=-K \partial \bar{u} / \partial z$, onde K é positivo e representa o coeficiente de troca turbulenta e (') e ( ') representam a média e o desvio da média, respectivamente. Apesar de a TCM ser utilizada com sucesso na Camada Limite Atmosférica, trabalhos teóricos (Moncrieff \& Miller, 1976; Moncrieff, 1981) e de análise de dados de radar coletados durante a experiência GATE sobre linhas convectivas (LeMone, 1983; LeMone et al., 1984) mostraram que esta aproximação não se verifica sempre. Nestes casos, o fluxo produz-se no mesmo sentido do cisalhamento, não favorecendo a redistribuição da quantidade de movimento.

O processo de análise utilizado para estudar o transporte de quantidade de movimento pela convecção organizada em linhas, consiste em considerar separadamente as componentes do movimento perpendicular (u) e paralelo (v) à linha convectiva (LC). Assim, separa-se as componente do vento em componentes médias e em perturbações, tais que: $u=\bar{U}+u^{\prime}$; $v=\bar{V}+v^{\prime}$ e $w=\bar{W}+w^{\prime}$. As componentes do vento médio $(\bar{U}, \bar{V}$ e $\bar{W})$ e suas perturbações (u', v' e w') são calculadas num sistema de coordenadas com o eixo x perpendicular à linha convectiva, positivo na direção de deslocamento do sistema, convenção adotada por LeMone \& Moncrieff (1994), ao analisar os fluxos verticais de quantidade de movimento de alguns sistemas convectivos de mesoescala. No presente caso, considerou-se o sistema de coordenadas representado na Fig. 3. Neste sistema a componente u perpendicular à linha convectiva, é positiva na direção de deslocamento da linha $\left(238^{\circ}-\mathrm{SW}\right)$ e a componente $\mathrm{v}$, paralela à linha, é positiva para $148^{\circ}$ (S-SE). Negligenciando as flutuações de densidade, o fluxo vertical total de quantidade de movimento perpendicu-

lar à linha convectiva $\bar{\rho} \overline{u w}$, é calculado usando a Eq.(2) e o fluxo vertical total de quantidade de movimento paralelo à linha convectiva $\bar{\rho} \overline{v w}$, é calculado usando a Eq.(3):

$$
\begin{aligned}
& \bar{\rho} \overline{u w}=\bar{\rho} \overline{u^{\prime} w^{\prime}}+\bar{\rho} \bar{U} \bar{W} \\
& \bar{\rho} \overline{u w}=\frac{\bar{\rho}}{N} \sum_{i=1}^{N} u_{i}^{\prime} w_{i}{ }_{i}+\bar{\rho} \bar{U} \bar{W} \\
& \bar{\rho} \overline{v w}=\bar{\rho} \overline{v^{\prime} w^{\prime}}+\bar{\rho} \bar{V} \bar{W} \\
& \bar{\rho} \overline{v w}=\frac{\bar{\rho}}{N} \sum_{i=1}^{N} v_{i}{ }_{i} w_{i}{ }_{i}+\bar{\rho} \bar{V} \bar{W}
\end{aligned}
$$

onde $\mathrm{N}$ é o número de pontos i em cada nível; $\bar{\rho} \bar{U} \bar{W}$ e $\bar{\rho} \bar{V} \bar{W}$ representam o fluxo vertical associado ao movimento médio; $\bar{\rho} \overline{u^{\prime} w^{\prime}}$ e $\bar{\rho} \overline{v^{\prime} w^{\prime}}$ representa ofluxo vertical perturbado de quantidade de movimento horizontal; $\bar{U}, \bar{V}$ e $\bar{W}$ representam a média do vento em cada plano vertical (de 0,5 a $15 \mathrm{~km}$ de altitude). A derivada vertical desses fluxos traduz a aceleração da velocidade horizontal correspondente.

Na literatura, o termo "downgradient" é utilizado se 
a TCM se verifica; no caso contrário, emprega-se o termo "upgradient" ou "countergradient" (LeMone et al., 1984). Neste trabalho definiremos os dois termos: "downgradiente" como redutor do gradiente e "upgradiente" como amplificador do gradiente.

\section{RESULTADOS E DISCUSSÕES}

Inicialmente foram analisados os resultados obtidos para a estrutura cinemática do SCM, com a aplicação do método EODD aos dados coletados pelos dois aviões WP-3D. A Fig. 2 mostra o campo de vento horizontal relativo composto e as isolinhas de refletividade nos níveis de $0,5,4,5$ e $7,5 \mathrm{~km}$, para o período 18:16-20:21UTC, que foi obtido conforme descrito na seção anterior.

Nos baixos níveis (Fig. 2a), a zona convectiva (SO da figura) é marcada por um fluxo convergente de oeste de mais de $10 \mathrm{~m} \cdot \mathrm{s}^{-1}$, que alimenta o sistema e mais particularmente as ascendências convectivas. Atrás deste fluxo, pode-se observar um fluxo divergente de oeste que se incorpora ao sistema pelo norte $(\mathrm{y}=0-30$ $\mathrm{km})$. Parte deste fluxo é desviada para o sul, atrás do fluxo que entra, e alimenta as subsidências convectivas. Uma outra parte, a mais larga é acelerada para trás dentro da região estratiforme (na direção NE) onde, por essência, a subsidência de ar ocorre. Essas são algumas características bem conhecidas dos SCM (Houze \& Betts, 1981; Chong et al., 1987; Roux, 1988).

Nas camadas intermediárias (Fig. 2b) e altas (Fig. 2c), o fluxo é principalmente de S-SO na região convectiva, com um aspecto divergente acima das células convectivas (a SO do domínio). Após a linha convectiva, este fluxo se incorpora ao sistema e mais especificamente à região estratiforme sofrendo deformações. Pode-se notar a rotação ciclônica marcada a NE do domínio, que desvia o fluxo para NO.

Para analisar o fluxo de ar relativo perpendicular à linha convectiva, foi feito um corte vertical ao longo da linha AA' da Fig. 2. Observa-se na parte superior da Fig. 4, à frente do sistema, um fluxo entrante ascendente, que é evacuado para trás em altitude e um fluxo descendente, atrás da linha convectiva dentro da região estratiforme. Pode-se notar também que a célula principal, localizada em $x=-25 \mathrm{~km}$ é precedida por uma célula mais nova $x=-85 \mathrm{~km}$, que estão associadas aos máximos de refletividade. Este sistema apresenta um fluxo de ar perpendicular à linha convectiva muito similar a características cinemáticas de linhas de instabilidade tropicais documentadas anteriormente (Houze, 1993). Essas características incluem, por exemplo, o forte fluxo dirigido para trás e para cima na troposfera, que é composto principalmente de ar que se originou nos baixos níveis a frente da LC, que alimenta a ascendência principal. Observa-se também que as subsidências convectivas são claramente separadas das subsidências de mesoescala. Estas últimas, na região estratiforme contribuem, em grande parte, para alimentar o fluxo dirigido para trás do sistema. Também pode-se notar a inclinação para trás dos núcleos de ascendência, mas não observa-se um fluxo de retorno principal, que é o agente pelo qual a região estratiforme realimenta a convecção, provavelmente associado à fase dissipativa do sistema analisado. Não observa-se também, a presença de uma corrente de densidade associada à subsidência convectiva, que pode ser explicado pela limitação da análise a níveis acima de $500 \mathrm{~m}$ de altitude. Ainda assim, pode-se constatar que a estrutura deste sistema é comparável àquela apresentada por LeMone et al. (1990) para um sistema convectivo caracterizado por um cisalhamento moderado perpendicular à linha convectiva.

Os movimentos verticais associados foram traçados sob a forma de isolinhas e são apresentados na Fig. 4 (painel inferior). Eles foram obtidos pela integração vertical da divergência associada ao campo de vento composto. Pode-se distinguir perfeitamente, nesta figura, as estruturas convectivas $(\mathrm{x}<-10 \mathrm{~km})$ orientadas verticalmente, das estruturas estratiformes mais alongadas atrás $(x>0 \mathrm{~km})$ orientadas horizontalmente, tanto para as ascendências como para as subsidências. Os picos de ascendência são encontrados em altitudes maiores e células de subsidência próximas a $\mathrm{x}=-35$ $25 \mathrm{~km}$, são observadas na região convectiva, em níveis mais baixos. Essas subsidências de baixos níveis induzem a ocorrência de precipitações convectivas, que são geralmente associadas a aguaceiros e tempestades de granizo e que alimentam o ar frio que se acumula abaixo da região convectiva, que forma a frente de rajadas dos SCM. Além das subsidências de baixos níveis pode-se observar subsidências de altitude, que segundo Houze (1993) tendem a ocorrer em ambos lados dos núcleos e ascendências. No presente caso isso pode ser visto em $(x=-45 \mathrm{~km}, \mathrm{y}=6 \mathrm{~km}) \mathrm{e}(\mathrm{x}=-5 \mathrm{~km}$, $\mathrm{y}=9 \mathrm{~km})$. Um outro caráter clássico deste sistema pode ser observado na Fig. 4 (painel inferior), onde os núcleos de ascendência são organizados verticalmente na região convectiva e horizontalmente na região estratiforme. A organização dos movimentos verticais perpendicular à linha convectiva confirma as características clássicas do sistema analisado, que pode ser observado na região estratiforme do sistema onde temse um perfil de velocidade vertical negativo nos primeiros $7 \mathrm{~km}$ e positivo acima deste nível. 

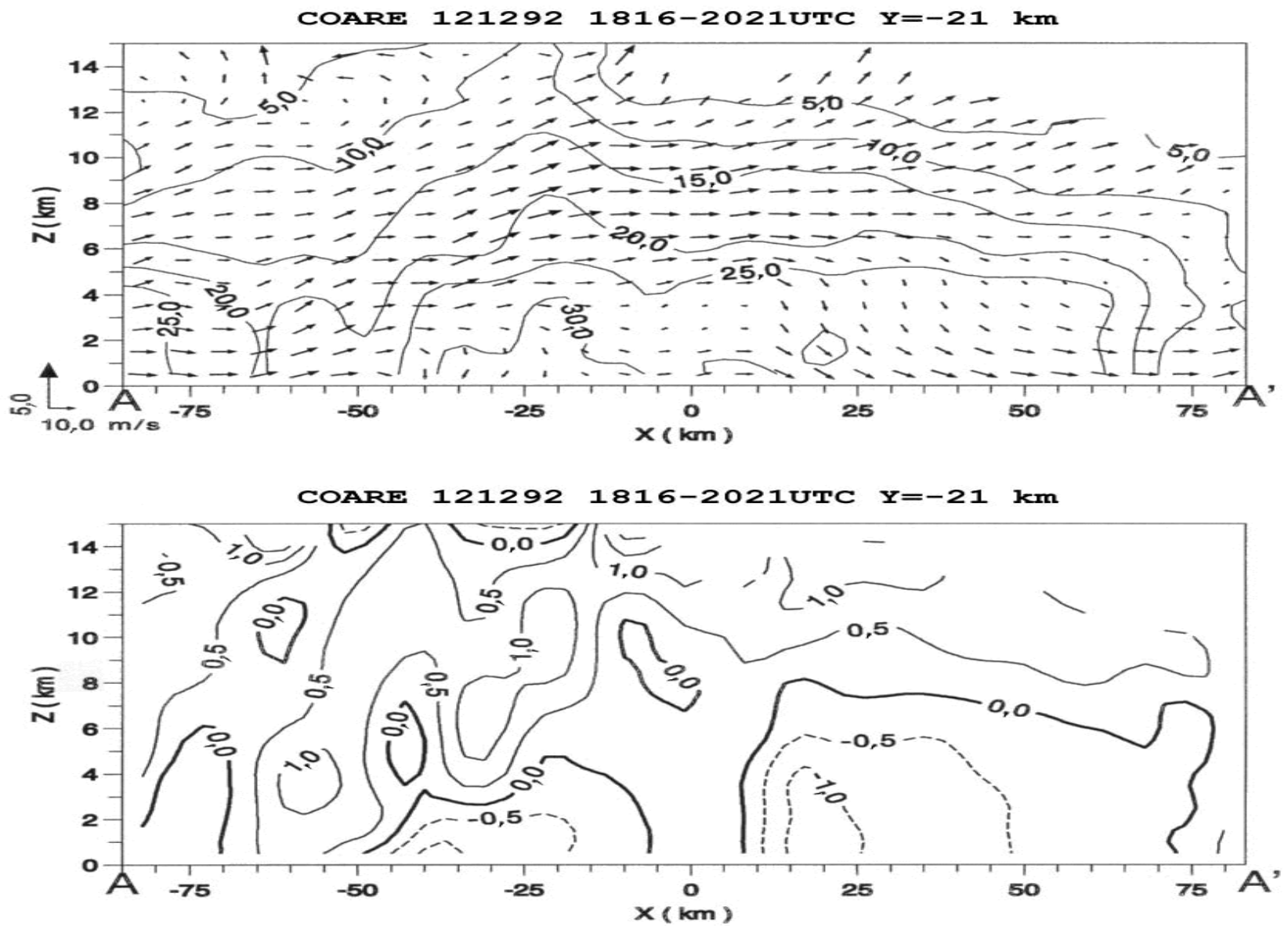

Figura 4- Parte superior: corte vertical do campo de vento relativo e da refletividade ao longo de AA' da Fig. 2. As coordenadas x representam a distância ao longo deste eixo, tendo como origem a projeção normal da origem do domínio sobre a seção considerada. Parte inferior: Isolinhas de velocidade vertical em $\mathrm{m} . \mathrm{s}^{-1}$.

Figure 4- Upper panel: vertical cross-section of relative flow and reflectivity along AA' of Fig. 2. $x$-coordinates refer to distance from normal projection of the origin to the considered section. Lower panel: vertical velocity contours in m.s. ${ }^{-1}$.

Em seguida foram analisados os resultados obtidos para os transportes de quantidade de movimento do SCM, que foram calculados utilizando o campo de vento composto mostrado na Fig. 2.

A Fig. 5 mostra os perfis de vento médio perpendicular $(\bar{U})$ e paralelo $(\bar{V})$ à linha convectiva e também o perfil de velocidade vertical média $(\bar{W})$. Na Fig. 6 os perfis de fluxo verticais de quantidade de movimento total e perturbado, perpendicular $(\bar{\rho} \overline{u w} \mathrm{e}$ $\left.\bar{\rho} \overline{u^{\prime} w^{\prime}}\right)$ e paralelo $\left(\bar{\rho} \overline{v w}\right.$ e $\left.\bar{\rho} \overline{v^{\prime} w^{\prime}}\right)$ à linha convectiva são apresentados. Enfim, a Fig. 7 mostra as acelerações, perpendicular e paralela à linha convectiva, que foram derivadas da divergência vertical dos fluxos de quantidade de movimento mostrados na Fig. 6. O perfil de velocidade vertical médio (Fig. 5) é positivo e apresenta um máximo numa altitude relativamente elevada $(11 \mathrm{Km})$, confirmando uma das característica observada durante TOGA-COARE (Lee \& Hildebrand, 1994; Smull et al., 1995).
O perfil para a componente $\mathrm{u}$ (perpendicular à linha convectiva) mostra valores mínimos nas camadas mais baixas e a $8 \mathrm{~km}$, que são separados por um máximo em 3,5 km (Fig. 5). Observa-se também neste perfil, um cisalhamento vertical de u positivo abaixo de $3 \mathrm{~km}$ e acima de $8 \mathrm{~km}$ e um cisalhamento vertical de u negativo nas camadas intermediárias. O fluxo vertical de quantidade de movimento horizontal perpendicular à linha convectiva $(\bar{\rho} \overline{u w})$ é negativo na troposfera com um mínimo em $8 \mathrm{~km}$, o que produz acelerações para frente (positiva) nas camadas baixas e para trás (negativo) em altitude (Fig. 7). A aceleração positiva produz uma diminuição do cisalhamento vertical do vento abaixo de $8 \mathrm{~km}$ e a aceleração negativa produz um fortalecimento do cisalhamento vertical do vento nos níveis mais altos. A comparação dos perfis de $\bar{\rho} \overline{u w}$ e $\bar{\rho} \overline{u^{\prime} w^{\prime}}$ (Fig.6) indica que a maior parte do transporte é devido ao fluxo médio. Comparado 

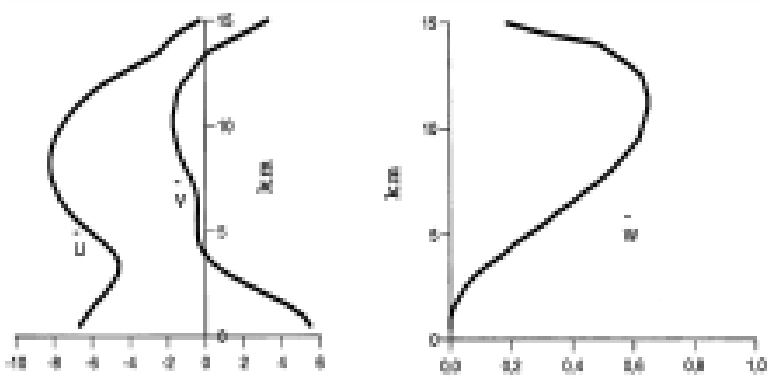

Figura 5- Perfis de vento médio perpendicular $(\bar{U})$ e paralelo $(\bar{V})$ à linha convectiva (esquerda) e da velocidade vertical média (direita).

Figure 5- Profiles of the mean wind components $u$ and $v$ in a $238^{\circ}$ oriented right-handed coordinate system (left panel), and of the mean vertical velocity (right panel).

os perfis de $\bar{U}$ e $\bar{\rho} \overline{u w}$ observa-se que o transporte de quantidade de movimento perpendicular à linha convectiva é redutor do gradiente (previsto pela TCM) abaixo de $3 \mathrm{~km}$ e acima de $8 \mathrm{~km}$ e amplificador do gradiente entre esses níveis. Em vários casos estudados (LeMone, 1983; LeMone et al., 1984; Matejka \& LeMone, 1990) também foi observado que o $\bar{\rho} \overline{u w}$ é negativo na troposfera, com um máximo absoluto nos níveis médios, associado a trajetória do fluxo de ar. A altura desse máximo absoluto corresponde aquela na qual a aceleração positiva dos baixos níveis muda para aceleração negativa acima (Fig. 7). Esse fluxo negativo é devido aos movimentos ascendentes convectivos originados abaixo de $1 \mathrm{~km}$, no fluxo de ar que entra no sistema (Fig. 4), e gera movimentos ascendentes que transportam quantidade de movimento da frente para trás do sistema. Esses autores também observaram, como no presente trabalho, que u é acelerado de trás para a frente do sistema (aceleração positiva) nos níveis mais baixos e da frente para trás (aceleração negativa) nos níveis mais altos. Segundo LeMone (1983) o transporte de quantidade de movimento se dá principalmente pela corrente de ascendências ("jump updraft"), que é acelerada para trás e para cima pela flutuabilidade. Os perfis de $\bar{\rho} \overline{u w}$ e $-\bar{\rho}^{-1} \partial(\bar{\rho} \overline{u w}) / \partial z$, obtidos neste trabalho, são muito similares aqueles preditos por Moncrieff (1992) e aqueles observados por LeMone \& Moncrieff (1994).

A comparação das Figs. 5 e 7 sugere que a aceleração líquida de $u$ atua no sentido de aumentar o cisalhamento vertical de $\mathrm{u}$, o que gera um transporte amplificador do gradiente. Segundo LeMone \& Moncrieff (1994), isso é comumente observado em bandas de convecção profunda e é causado pelo trans- porte vertical de quantidade de movimento horizontal perpendicular à linha convectiva pelo fluxo de ar organizado.

O perfil para a componente $\mathrm{v}$, paralela a linha convectiva (Fig. 5), apresenta dois mínimos em 4 e 10 $\mathrm{km}$. Observa-se também nesse perfil um cisalhamento negativo abaixo de $10 \mathrm{~km}$, mais pronunciado nos 5 primeiros $\mathrm{km}$. O fluxo vertical de quantidade de movimento horizontal paralelo à linha $(\bar{\rho} \overline{v w})$ é positivo abaixo de $6,5 \mathrm{~km}$ e negativo acima deste nível, com valores extremos ocorrendo respectivamente em $3 \mathrm{e}$ $9,5 \mathrm{~km}$. A comparação do fluxo total com o fluxo perturbado mostra que o fluxo perturbado tem uma grande contribuição abaixo de $6 \mathrm{~km}$. O fluxo positivo tem um efeito redutor do gradiente enquanto que o fluxo negativo é amplificador do gradiente entre 6,5 e 9,5 km e redutor do gradiente acima de $9,5 \mathrm{~km}$. O fluxo perturbado $\bar{\rho} \overline{v^{\prime} w^{\prime}}$ é positivo e tem uma contribuição similar abaixo de $6 \mathrm{~km}$. Os fluxos médio e perturbado produzem acelerações negativas nas camadas baixas e acelerações positivas nas camadas médias, contribuindo assim para diminuir o cisalhamento do vento paralelo. A divergência do fluxo de $\mathrm{v}$ tende a reduzir o cisalhamento vertical de $v$, gerando um transporte redutor do gradiente. Estes resultados são similares aqueles obtidos em estudos precedentes (LeMone, 1983; LeMone et al, 1984; LeMone \& Jorgensen, 1991), onde o $\bar{\rho} \overline{\nu w}$ foi bem descrito qualitativamente pela TCM com o cisalhamento vertical ao longo da linha sendo reduzido de seu valor original.

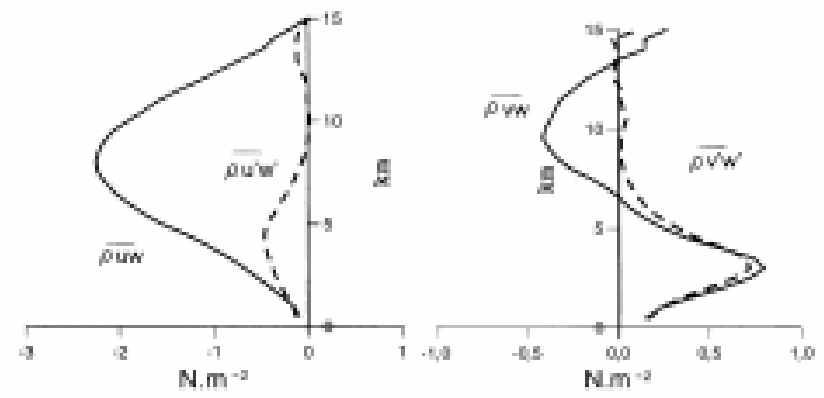

Figura 6 - Perfis dos fluxos verticais de quantidade de movimento horizontal total $(\bar{\rho} \overline{u w}$ e $\bar{\rho} \overline{v w})$ e perturbado $\left(\bar{\rho} \overline{u^{\prime} w^{\prime}}\right.$ e $\bar{\rho} \overline{v^{\prime} w^{\prime}}$ ), perpendicular (esquerda) e paralelo (direita) à linha convectiva.

Figure $6-u$ and $v$ momentum flux profiles total $(\bar{\rho} \overline{u w}$ and $\bar{\rho} \overline{v w}$ ) and perturbed $\left(\bar{\rho} \overline{u^{\prime} w^{\prime}}\right.$ and $\bar{\rho} \overline{v^{\prime} w^{\prime}}$ ), for the linenormal (left) and line-parallel (right) 


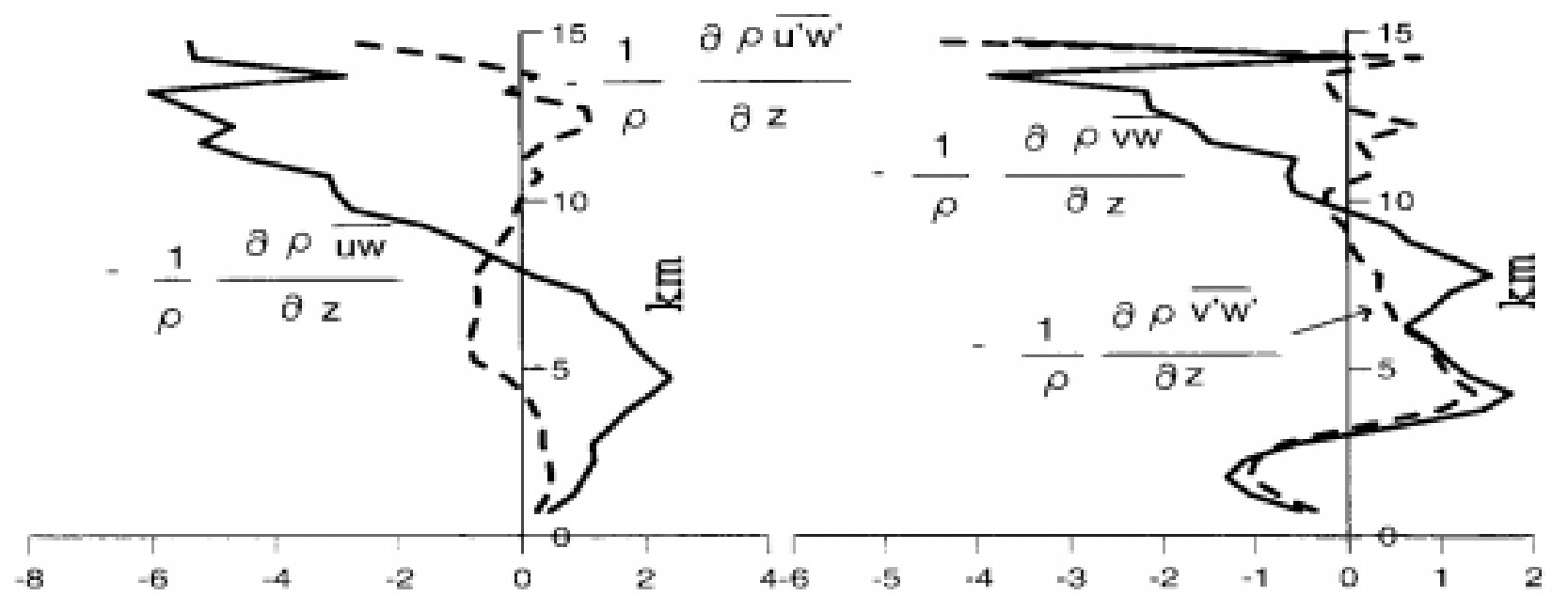

Figura 7- Acelerações, perpendicular e paralela à linha convectiva, que foram derivadas da divergência vertical dos fluxos de quantidade de movimento mostrados na Fig. 6.

Figure 7 -Line-normal (left panel) and line-parallel (right panel) accelerations as deduced from the vertical divergence of the fluxes in Fig. 6.

\section{CONCLUSÕES}

O sistema convectivo do dia 12 de dezembro de 1992 foi analisado a partir de dados de radares Doppler embarcados nos aviões P3 da NOAA. A restituição do campo de vento 3D foi obtida utilizando o método EODD. Um campo de vento composto foi obtido para fornecer uma visão global e sintética do sistema. A comparação dos resultados obtidos neste trabalho com aqueles de trabalhos precedentes mostram o caráter clássico da estrutura cinemática do sistema observado, com um fluxo ascendente na frente do sistema que transporta o ar das camadas inferiores para o alto e um fluxo descendente atrás da linha convectiva.

Os resultados relativos aos transportes de quantidade de movimento mostram que a forma dos perfis verticais de fluxo de quantidade de movimento horizontal perpendicular à linha convectiva $(\bar{\rho} \overline{u w})$ e da divergência vertical do fluxo de quantidade de movimento horizontal perpendicular à linha convectiva

$\left(-\bar{\rho}^{-1} \partial(\bar{\rho} \overline{u w}) / \partial z\right.$ do sistema convectivo do dia 12/12/92, apresentam similaridades com aqueles preditos por Moncrieff (1992) e com aqueles observados por LeMone \& Moncrieff (1994). Algumas similaridades observadas foram: (i) o fluxo $\bar{\rho} \overline{u w}$ obtido foi negativo na troposfera, com um mínimo nos níveis médios; (ii) a componente u é acelerada para frente do sistema nos níveis baixos e para trás nos níveis mais altos; (iii) a aceleração de $u$ atua no sentido de aumentar o cisalhamento vertical de u, gerando um transporte que é amplificador do gradiente.

Para a componente paralela à LC (v), a aceleração de $\mathrm{v}$, que está associada ao transporte de quantidade de movimento pela divergência vertical do fluxo de v, é negativa nos baixos níveis e positiva nas camadas médias, contribuindo assim para diminuir o cisalhamento do vento paralelo. Essa configuração gera um transporte redutor do gradiente e é bem descrita qualitativamente pela TCM, conforme já foi observado anteriormente.

Os resultados apresentados neste trabalho representam mais um passo para a compreensão dos sistemas convectivos que ocorrem sobre a região do oceano Pacífico oeste. Outras investigações são necessárias para avaliar o impacto sobre o ambiente, envolvendo a análise da dinâmica e da termodinâmica associada ao sistema convectivo do dia 12 de dezembro de 1992.

\section{REFERÊNCIAS}

BROWNING, K.A. \& WEXLER, R. - 1968 - The determination of kinematic properties of a wind field using Doppler radar. J. Appl. Meteor., 7: 105-113.

CAMPOS, C.R.J. - 1996 - Etude des Sistèmes Précipitants Convectifs par Radar Doppler Aéroporté: Aplication a un cas de TOGA-COARE. Tese de doutorado, UPSFrança, p. 146.

CHEN, S.S., HOUZE, R. A. \& MAPES, B.E. - 1996 Multiscale variability of deep convection in relation to large-scale circulation in TOGA-COARE. J. Atmos. Sci., 53: $1380-1409$.

CHONG, M. \& CAMPOS, C.R.J. - 1996 - Extended overdetermined dual-Doppler formalism in sinthesizing airborne Doppler radar data. J. Atmos. Oceanic Technol., 13: 581-597.

CHONG, M. \& TESTUD, J. - 1983 - Three-dimensional wind 
field analysis from dual-Doppler radar data. Part III: The boundary condition: an optimum determination based on variational concept. J. Climate Appl. Meteor., 22: 1227-1241.

CHONG, M., AMAYENC,P., SCIALOM, G. \& TESTUD, J. 1987 - A tropical squall line observed during the COPT81 experiment in west Africa. Part 1: kinematic structure inferred from dual-Doppler radar data. Mon. Wea. Rev., 115: 670-694.

CRESSMAN, G.W. - 1959 - An operational objective analysis system. Mon. Wea. Rev., 87: 367-374.

HOUZE, R. A. - 1993 - Mesoscale convective systems. In Cloud dynamics. Academic Press, Inc., vol. 53: 334404.

HOUZE, R. A. \& BETTS, A.K. - 1981 - Convection in GATE. Rev. Geophys. Space Phys., 19: 541-576.

JORGENSEN, D.P. \& DUGRANRUT, J.D. - 1991 - A dualbeam technique for deriving wind fields from airborne Doppler radar. Proceedings $25^{\text {th }}$ Radar Meteorology Conf., Amer. Meteor. Soc., 458-461, Paris.

LEE, W.-C. \& HILDEBRAND, P.H. - 1994 - Comparison on the kinematic structures of a TOGA-COARE squall line using data from NCAR ELDORA annd two NOAA P3s airborne Doppler radars. Proceedings 6th Conf. on Mesoscale Processes, Amer. Meteor. Soc., 105-108, Portland.

LEMONE, M.A. - 1983 - Momentum flux by line of cumulonimbus. J. Atmos. Sci., 40: 1815-1835.

LEMONE, M.A. \& JORGENSEN, D.P. - 1991- Precipitation and kinematic structure of an oceanic mesoscale convective siystem. Part II: Momentum transport and generation. Mon. Wea. Rev., 119: 2638-2653.

LEMONE, M.A. \& MONCRIEFF, M.W. - 1994 - Momentum and mass transport by convective bands: comparison of highly idealized dynamical models to observations. J. Atmos. Sci., 51: 281-304.

LEMONE, M.A., BARNES, G.M. \& ZIPSER, E.J. - 1984 Momentum fluxes by lines of cumulonimbus over the tropical oceans. J. Atmos. Sci., 41: 1914-1932.
LEMONE, M.A., FANKHAUSER, J.C. \& MATEJKA, T. 1990 - Momentum generation and redistribution in convective bands: what have we learned? Proceedings 4th Conf. on Mesoscale Processes, Amer. Meteor. Soc., 198-199.

MATEJKA, T. \& LEMONE,M.A. - 1990 - The generation and redistribution of momentum in a squall line. Proceedings Fourth Conf. On Mesoscale Processes, Amer. Met. Soc., 196-197, Boulder.

MONCRIEFF, M.W. - 1981 - A theory of organized steady convection and its transports properties. Quart. J. Roy. Meteor. Soc., 107: 29-50.

MONCRIEFF, M.W. - 1992 - Organised mososcale convective systems: archetypal dynamical models, mass and momentum flux theory, and parametrization. Quart. J. Roy. Meteor. Soc., 118: 819-850.

MONCRIEFF, M.W. \& MILLER, M.J. - 1976 - The dynamics and simulation of tropical cumulus and squall line. Quart. J. Roy. Meteor. Soc., 102: 373-394.

ROUX, F. - 1988 - The west-african squall line observed on 23 june 1981 during COPT81. Kinematics and thermodynamics of the convective region. J. Atmos. Sci., 45: 406-426.

REDELSPERGER, J.L., JABOUILLE,P. \& LAFORE, J.-P 1995 - Numerical studies of convective organization observed during TOGA-COARE. Proceedings 21st Conf. on Hurricanes and Tropical Meteorology, 582-584, Miami.

SMULL, B.F., JORGENSEN, D.P. \& MATEJKA, T.J. - 1995 - Airborne Doppler radr and in situ measurements of a slow-moving convective band observed during TOGACOARE. Proceedings 21st Conf. on Hurricanes and Tropical Meteorology, 428-430, Miami.

VELDEN, C.S. \& YOUNG, J.A. - 1994 - Satellite observations during TOGA-COARE: large-scale descriptive overview. Mon. Wea. Rev., 122: 2426-2441.

WEBSTER, P. J. \& LUKAS, R. - 1992 - TOGA-COARE: The coupled ocean-atmosphere response experiment. Bull. Amer. Meteor. Soc., 73: 1377-1416. 


\section{7th International Congress of the Brazilian Geophysical Society}

The Technical Program will include oral and poster sessions on exploration and environmental applications of seismic, potential fields, borehole, nuclear and geologic methods, as well as solid earth and space geophysics, instrumentation and case histories. The deadline for expanded abstracts is March 31, 2001 (maximum four pages including figures). For Abstract Kits with detailed instructions, contact the 7th CISBGf Office:

\section{7th CISBGf Office}

Instituto de Física da UFBa

Campus Universitário de Ondina

40210-340 Salvador, Bahia - Brasil

Phone-Fax : +55(71)235-0002

E-mail: cisbgf@sbgf.org.br

The Salvador 2001 International Congress is sponsored by the Brazilian Geophysical Society (SBGf), the Society of Exploration Geophysicists (SEG), the European Association of Geoscientists and Engineers (EAGE) and the Latin American Geophysical Union (ULG). Whether your interest is in the technical conference or in the exhibition, you will find in Salvador the perfect venue to update your knowledge or do business.

Exhibition: Salvador 2001 will offer a truly outstanding marketing opportunity for Latin America. Special invitations are extended to state owned and private companies to attend and participate. Companies wishing to receive additional exhibitor information should contact the SBGf Business Office.

Technical Conference - Whether going deep into the ground or probing into outer space, geophysics is continually revealing a new wealth of understanding. Geophysicists must continuously follow the new advances in their fields of study and Salvador 2001 is the ideal place to see the recent developments, meet old friends and make new ones.

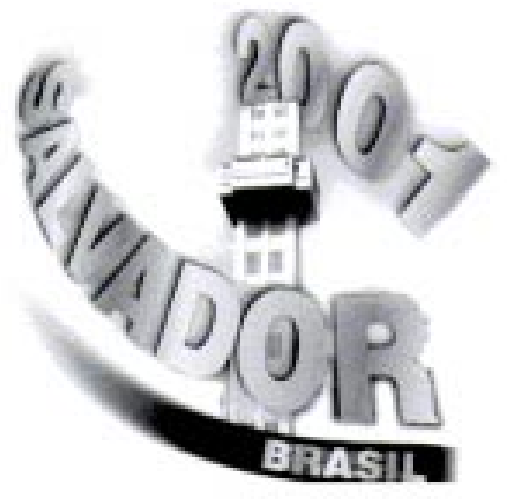

SALVADOR 2001: COME TO SEE WHAT'S NEW IN BRASIL BALL FOR PAPERS

$7^{a}$ Congresso Internacional da Sociedade Brasileira de Geofisica 7th International Congress of the Brazilian Geophysical Society Salvador, Brasil 